\title{
Infiltrative lobular breast carcinoma presenting initially as an orbital metastatic lesion
}

\begin{abstract}
The incidence of orbital metastases is estimated to be 1-2 per year in large centers. The commonest orbital metastasis is from breast cancer, which tends to occur late followed by lung cancer which tends to occur relatively early in addition to a proportion of metastatic lesions from unknown primary sites. The metastatic breast lesions to the orbit can be discovered as late as 20-25 years following the identification of the primary tumor. However, the orbital metastatic lesion can be the initial manifestation in a small number of patients. We are presenting a 48-year-old female patient where the diagnosis of her lobular breast carcinoma was made after her initial presentation to the ophthalmic service because of diplopia. Her clinical course, histopathological findings of her orbital lesion as well as the radiological findings are described with extensive review of the most recent literature.
\end{abstract}

Keywords: orbital metastasis, breast lobular carcinoma
Volume 2 Issue 6 - 2015

\author{
Hind Alkata,' Yerena Muiños Diaz, ${ }^{2,3}$ Alicia \\ Galindo Ferreiro, ${ }^{2}$ Sahar M EIKhamary ${ }^{4}$ \\ 'Department of Ophthalmology, King Saud University, Saudi \\ Arabia \\ ${ }^{2}$ Department of Oculoplastics, King Khaled Eye Specialist \\ Hospital, Saudi Arabia \\ ${ }^{3}$ Department of Ophthalmology, Hospital Nuestra Señora \\ defátima, Spain \\ ${ }^{4}$ Department of Diagnostic Radiology, King Khaled Eye Specialist \\ Hospital, Saudi Arabia
}

\section{Correspondence: Hind Alkatan, Department of \\ Ophthalmology, College of Medicine, King Saud University, Riyadh, KSA, Saudi Arabia, Tel +966504492399, \\ Emailhindkatan@yahoo.com}

Received: July 31, 2015 | Published: August 04, 2015
Abbreviations: EOM, extra-ocular motility; MRI, magnetic resonance imaging; CT, computed tomography; PAS, periodic acid schiff; IHC, immunohistochemical; ER, estrogen receptor; PR, progesterone receptor; FNA, fine needle aspiration; ILBC, infiltrating lobular breast cancer

\section{Introduction}

Metastatic breast cancer to the ocular structures has a wide range of ophthalmic and neurologic manifestations including orbital, neural, choroidal and brain involvement in order of frequency. ${ }^{1}$ The incidence of orbital metastases as a whole is estimated to be 1-2 per year in large centers. ${ }^{2}$ The commonest orbital metastasis is from breast cancer, which tends to be as late as 20-25 years following the identification of the primary tumor. ${ }^{3}$ However, the orbital metastatic lesion can be the initial manifestation in a small number of patients such as in our case, which we are presenting.

\section{The case}

A 48-year-old premenopausal female presented to the ophthalmologist complaining of diplopia over the last 9 months. Her external examination revealed mild right Enophthalmos with limitation of extra-ocular motility (EOM). Her visual acuity was $20 / 20$ on the right and $20 / 25$ on the left with normal ocular pressure. Her Hertel measurements at base of 95 were 14 on the right and 17 on the left. Neuro-ophthalmic examination revealed normal cranial nerves and no evidence of ptosis or palsy. The medical history revealed positive family history of breast cancer. She was also diabetic on oral hypoglycemic for the last 7 years.

Her magnetic resonance imaging (MRI) at presentation Figure $1 \mathrm{~A} \& \mathrm{~B}$ showed right small soft tissue mass between the optic nerve and the medial rectus muscle. Further imaging studies by computed tomography (CT) and MRI showed significantly enlarging orbital lesion over one year (Figure $1 \mathrm{C}-\mathrm{H}$ ). However, there was no extension to the brain.

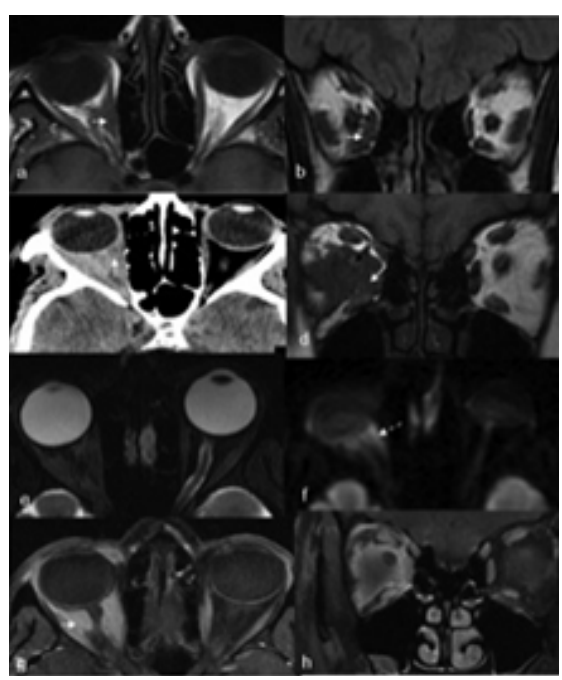

Figure I (A-H):Initial Magnetic Resonance (a:Axial and b:CoronalTI -weighted Images) in 2013 showing a small soft tissue mass between the optic nerve and the medial rectus muscle (white arrow). (2C) Non contrast Computerized Tomography (CT) scan done in 2014 showing significant increase in the size of the lesion filling the RT retro-bulbar area. (2D-H) coronal TI, Axial T2, the retro-bulbar area infiltration with restricted pattern of the lesion on Diffusionweighted MRI image $(f)$ indicating high degree of cellularity, malignancy (white dashed arrow)with intense enhancing pattern on post contrast fat suppressed images that encircling the optic nerve which appears as a black dot against the whitish diffusely enhancing mass $(\mathrm{G}-\mathrm{H})$. 
Histopathologic examination of right orbital tumor incisional biopsy showed proliferation of single pleomorphic cells and small clusters of cells with moderate nuclear pleomorphism and occasional prominent nucleoli infiltrating the orbital fibro-adipose tissue (Figure 2A) The cells showed intra-cytoplasmic lumina which showed mucin by Alcian blue and periodic acid Schiff (PAS) stains (Figure 2B). Some tumor cells showed cord configuration and were embedded in dense fibrous stroma.

The immunohistochemical (IHC) staining revealed positive tumor cells for epithelial markers (cytokeratin and epithelial membrane antigen) as in (Figure 2C\&D). The cells were negative for CD3, CD20 and CD68. The initial impression was a metastatic adenocarcinoma therefore IHC staining for gross cystic disease fluid protein -15 (GCDFP-15) was requested and the tumor cells were positive (Figure $2 \mathrm{E} \& \mathrm{~F})$. Taking into consideration that this tumor is deeply seated in the orbit, the GCDFP-15 positivity and the family history of breast cancer, our diagnosis was highly suspicious of a metastatic breast carcinoma.

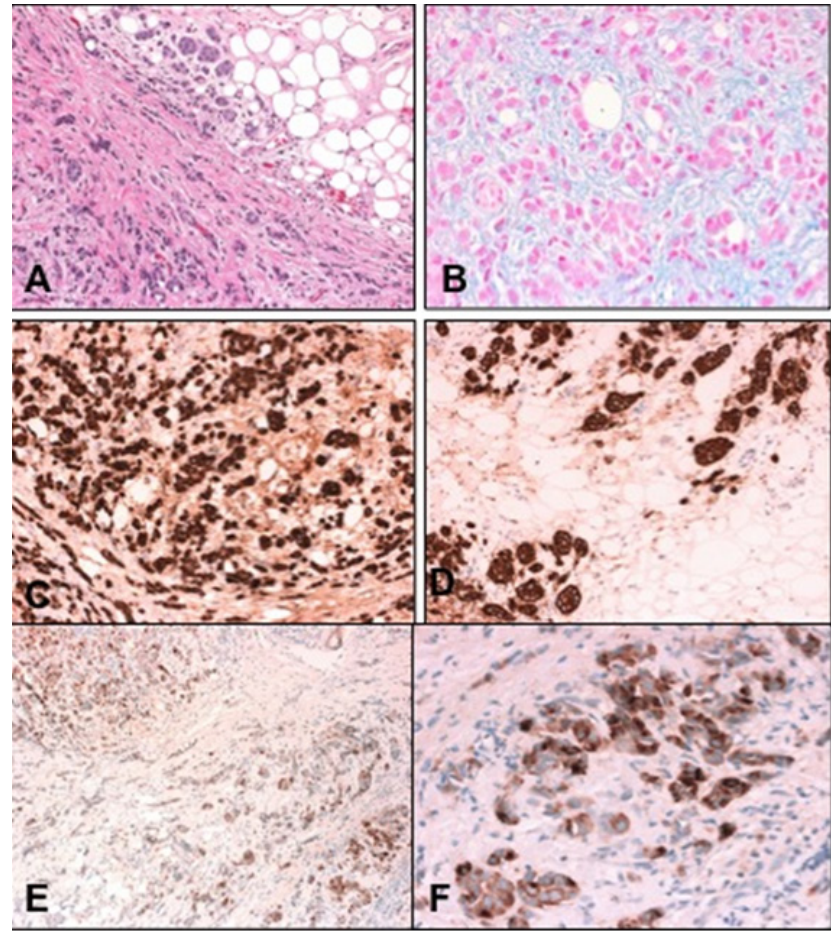

Figure 2 (A) The right orbital biopsy showing small clusters of tumor cells infiltrating fat (Original magnifications X200 Hematoxylin \& Eosin). (B) The clusters of tumor cells with mucinous secretions (Original magnification X400 Alcian Blue). (C) The positively staining tumor cells within fibrous stroma (Original magnification X200 CytoK AEI/AE3). (D) The epithelial tumor cells infiltrating orbital fat (Original magnification X200 EMA). (E) The tumor cells within the fibrous stroma showing positive staining (Original magnification XI00 GCDFP-I5). (F) Higher magnification of the positively stained tumor clusters (Original magnification X400 GCDFP-I5).

The patient was referred for mammogram, which showed right breast well-defined dense lesion with speculated margins measuring $3.7 \times 3.0 \mathrm{~cm}$ highly suspicious for malignancy. In addition the rounded dense axillary lymph nodes on the right side (Figure $3 \mathrm{~A} \& \mathrm{~B}$ ). Her surgical oncology clinical examination 4 months after the biopsy revealed a palpable right breast mass measuring $7 \times 6 \mathrm{~cm}$, mobile, not attached to skin or chest wall. The histopathology of her right breast mass showed invasive lobular carcinoma grade1, LVI was positive, Estrogen Receptor (ER) strongly positive $>80 \%$. Progesterone Receptor (PR) strongly positive $>90 \%$. Her-2 was negative with Ki67 of $10 \%$. Surprisingly another smaller breast mass was found in the contralateral breast for which biopsy was performed and the result was invasive lobular carcinoma grade 1 with ER strongly positive in $80 \%$, PR moderately positive in $10 \%$ and Her- 2 negative.

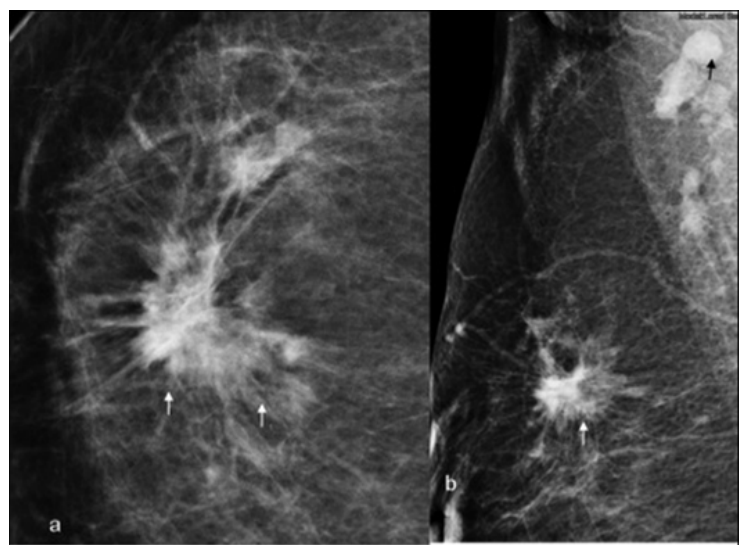

Figure 3 Cranio-caudal. (A) Medio-lateral. (B) mammogram views of the right breast showing a multifocal infiltrative whiskery lobular carcinoma (white arrows) with a rounded dense mass representing an involved axillary lymph node (black arrow).

Her systemic workup showed multiple sclerotic bone lesions so she was finally diagnosed as stage IV breast cancer $T_{3}, N_{1}, M_{1}$. The decision was to place her on Tamoxifen and Zometa with possible plan for proton therapy in Germany. She remained stable for 3 months following her definitive diagnosis.

\section{Discussion}

Metastatic breast cancer is the commonest primary tumor metastasizing to ocular structures in general. Bullock et al. ${ }^{1}$ in 1980 studied the wide range of ophthalmic manifestations of this disease, which has included orbital metastatic lesions, cranial nerve involvement, Horner's syndrome, choroidal involvement then brain involvement in order of frequency.

In regard to the orbital metastasis, it has been found that breast cancer is the commonest primary site in $(55-60 \%)$ and tends to occur late followed by lung cancer in (16-20\%) of orbital metastasis and it tends to occur relatively early. Another significant proportion $(10-30 \%)$ of orbital metastasis is from an unknown primary site. ${ }^{4-6}$ In a major recent review by Raap in 2015 on distant breast cancer metastases to the orbit, the incidence of histologically confirmed breast orbital metastases is estimated to be 1-2 cases per year at large clinical ophthalmic centers. ${ }^{2}$ Another interesting observation is that the latency of orbital metastasis from breast cancer might reach up to 20-25 years after the primary tumor is identified. ${ }^{3}$ However orbital metastasis in breast cancer can be the presenting manifestation in a small percentage of patients such as in our case. ${ }^{1}$

Following the definitive diagnosis, our patient declared a suspicious lesion in a previous right side mammogram 1 year prior to her ophthalmic presentation but the fine needle aspiration (FNA) at that time was negative. She also noticed the Enophthalmos for 9 months but did not seek ophthalmic consultation until she developed the diplopia. The Enophthalmos is usually related to a metastatic 
scirrhous breast carcinoma but the lobular variant can sometimes produce sclerosis leading as well to Enophthalmos. Shields et al. ${ }^{7}$ also reported Enophthalmos in relation to a case of lobular carcinoma with orbital metastasis and false negative mammogram and physical examination. They commented that this is occasionally noted if the lobular carcinoma remained in situ with no clinically apparent invasion. ${ }^{7,3}$ On the other hand metastatic scirrhous adenocarcinoma of the breast can present as proptosis. ${ }^{8}$ The enophthalmos, especially when associated with diplopia (as in our case) or motility problem should attract the attention to a metastatic lesion even without radiologic evidence of an orbital mass. ${ }^{9}$ Solari et al..$^{10}$ has reported other less common ophthalmic manifestations such as neurotrophic keratitis and it attracted the attention to a small breast cancer metastatic nodule at the left superior orbital rim.

Bilateral orbital metastases from breast carcinoma have been also reported with a rate of $20 \% .^{11,12}$ If the involvement is infiltrative in nature, it can masquerade as an orbital inflammatory syndrome. Therefore, when "orbital pseudo tumor" is diagnosed clinically in an adult female patient, thorough medical history must be obtained as well as careful ophthalmic and systemic examination. ${ }^{12,13} \mathrm{Kim}$ and Grossniklaus ${ }^{14}$ reported extensive bilateral orbital metastases of lobular breast carcinoma filling both orbits from the eyelids to the apex. Raap et al. ${ }^{2}$ in his most recent paper reported the high frequency of infiltrating lobular breast cancer (ILBC) in patients with orbital metastases thus representing the cancer solo type which has the highest prevalence among reported orbital metastases. This further confirms the special metastatic behavior of this tumor. The dispersed histologic pattern of ILBC is thought to be related to its tumor biology, possibly involving the absence of E-Cadherin adhesion molecule in tumor cells, but other factors explaining this metastatic potential are still unclear. ${ }^{2,15}$

Orbital imaging with CT or MRI is helpful in guiding the biopsy in order to determine the appropriate surgical approach and to minimize injury to vital orbital structures. Almost all orbital metastases present as irregularly shaped masses on non-contrast CT, iso-dense to muscle, and usually hypo-intense to fat on T1-weighted images and hyperintense to fat on T2WI. MRI shows some degree of enhancement with contrast agents. The combined involvement of the orbit and adjacent structures, such as the para nasal sinuses, is a rare condition revealed by imaging studies. ${ }^{16}$ Tumor pattern can vary from diffusely infiltrative with obscured anatomical landmarks to a focal welldefined mass. ${ }^{17,18}$ Scirrhous (fibrotic) breast cancers are unique in their ability to produce enophthalmos and ophthalmoplegia. When orbital bone is involved, metastatic breast carcinoma tends to be destructive in nature rather than hyperostotic. ${ }^{19}$ In our case, the CT axial image demonstrated diffuse infiltration of the right inferior rectus, orbital fat, and eyelid soft tissue without bony involvement.

The prognosis following orbital metastasis is generally poor and the treatment modalities include radiotherapy, chemotherapy and hormonal treatment-such as in our case and good responses have been reported ${ }^{20}$ owing to the late presentation of our patient, palliative therapy with Tamoxifen was initiated. There might be possibility that the diagnosis of her primary ILBC on the right side was missed in her ophthalmic manifestation was considered in our report as the initial presentation of her primary tumor which was eventually found to be bilateral.

\section{Conclusion}

We are presenting a case of ILBC who presented to the ophthalmic service with enophthalmos and diplopia prior to the discovery of her primary breast cancer. Her late presentation and the advanced stage of her tumor affected the decision for palliative therapy. Health awareness regarding breast cancer and the availability of breast cancer screening programs through the health care facilities in our community is needed to allow earlier diagnosis in such cases.

\section{Funding}

None.

\section{Acknowledgments}

None.

\section{Conflicts of interest}

The authors have no conflicts of interest to declare.

\section{References}

1. Bullock JD, Yanes B. Ophthalmic Manifestations of Metastatic Breast Cancer. Ophthalmology. 1980;87(10):961-973.

2. Raap M, Antonopoulos A, Dammrich M, et al. High Frequency of Lobular Breast Cancer in Distant Metastases to the Orbit. Cancer Med. 2015;491:104-111.

3. Reeves D, Levine MR, Lash R. Nonpalpable Breast Carcinoma Presenting as orbital Infiltration: Case Presentation and Literature Review. Ophthal Plast Reconstr Surg. 2001;18(1):84-88.

4. Freedman MI, Folk JC. Metastatic Tumors to the Eye and Orbit. Patient Survival and Clinical Characteristics. Arch Ophthalmol. 1987;105:12151219

5. Font RL, Ferry AP. Carcinoma Metastatic to the Eye and orbit III. A Clinicopathologic Study of 28 Cases Metastatic to the Orbit. Cancer. 1976;38(3):1326-1335.

6. Fahmy P, Heegard S, Jensen OA, et al. Metastases in the ophthalmic region on Denmark 1969-98. A Histopathological Study. Acta Ophthalmol Scand. 2003;81(1):47-50.

7. Shields CL, Stopyra GA, Marr BP, et al. Enophthalmos as Initia Manifestation of Occult, Mammogram-Negative Carcinoma of the breast. Ophthalmic Surg Laser Imaging. 2004;35(1):56-57.

8. Jacobs M, Benger R. Metastatic Breast Carcinoma of the Orbit. Aust NZ J Ophthalmol. 1989;17(4):357-361.

9. Chang BY, Cunniffe G, Hutchinson C. Enophthlmos associated with primary Breast Carcinoma. Orbit. 2002;21(4):307-310.

10. Solari HP, Ventura MP, Cheema DP,et al. Orbital Metastasis from Breast Carcinoma Presenting as neurotrophic Keratitis. Can J Ophthalmol. 2006;41(1):93-96.

11. Hutchison DS, Smith TR. Ocular and Orbital Metastatic Carcinoma. Ann Ophthalmol. 1979;11(6):869-873.

12. Toller KK, Gigantelli JW, Spalding MJ. Bilateral Orbital Metastases from Breast Carcinoma. A Case of False Pseudotumor. Ophthalmology. 1998;105(10):1897-1901.

13. Murthy R, Gupta A, Hedge S, et al. Bilateral Multiple Extraocular Muscle metastasis from Breast Carcinoma. Indian $J$ Ophthalmol. 2011;59(5):381-382.

14. Kim HJ, Wojno TH, Grossniklaus H. Atypical Bilateral Orbital Metastases of Lobular Breast Carcinoma. Ophthalmol Plast Reconstr Surg. 2012;28(6):142-143.

15. Tomizawa Y, Ocque R, Ohori NP. Orbital Metastasis as the Initial Presentation of Invasive Lobular Carcinoma of Breast. Intern Med. 2012;51(12):1635-1638. 
16. Razek AA, Elkhamary S, Mousa A. Differentiation between benign and malignant orbital tumors at 3-T diffusion MR-imaging. Neuroradiology. 2011;53(7):517-522.

17. Wickremasinghe S, Dansingani KK, Tranos P, et al. Ocular presentations of breast cancer. Acta Ophthalmol Scand. 2007;85(2):133-142.

18. Surace D, Piscioli I, Morelli L, et al. Orbital metastasis as the first sign of "Dormant" breast cancer dissemination 25 years after mastectomy. Jpn J Ophthalmol. 2008;52(5):423-425.
19. Kadivar M, Joulaee A, Kashkouli MB, et al. Orbital metastasis as the first presentation of non-palpable invasive lobular carcinoma of the breast. Breast J. 2006;12(1):75-76.

20. Saitoh A, Amemiya T, Tsuda N. Metastasis of Breast Carcinoma to Eyelid and Orbit of a Postmenopausal Woman: Good Response to Tamoxifen Therapy. Ophthalmologica. 1997;211(6):362-366. 